\title{
A comparison of bolus injection of landiolol versus oral administration of propranolol before cardiac computed tomography
}

Yoshitaka Nakamura ${ }^{2+}$, Kyohei Yamaji ${ }^{1 *+}$, Tatsunori Saho ${ }^{2}$, Zyousin Matsuzaki $^{2}$, Itsuo Yuda ${ }^{2}$, Yoshimitsu Soga ${ }^{1}$, Shinichi Shirai ${ }^{1}$, Kenji Ando ${ }^{1}$ and Masakiyo Nobuyoshi ${ }^{1}$

\begin{abstract}
Heart rate (HR) reduction is essential to achieve good image quality for cardiac computed tomography (CCT). We evaluated the efficacy of a bolus injection of landiolol, an ultra-short acting $\beta$-blocker, without the administration of oral $\beta$-blocker to reduce HR prior to CCT. We enrolled 678 consecutive patients who underwent CCT from December 2011 to March 2012 and divided them into three groups, which were a propranolol group ( $n=277)$, a low-dose landiolol group $(n=188)$, and a high-dose landiolol group $(n=213)$. Patients in the propranolol group received oral propranolol (10-20 mg) prior to CCT. Patients in the low-dose and high-dose landiolol groups were administered a bolus injection of landiolol $(0.125 \mathrm{mg} / \mathrm{kg}$ ), while the high-dose group received an additional $3.75 \mathrm{mg}$ of landiolol if the baseline HR was $\geq 75 / \mathrm{min}$. Although the average HR was significantly lower in the propranolol group $(61.6 \pm 8.0 / \mathrm{min})$ than in the low-dose landiolol group $(64.1 \pm 7.4 / \mathrm{min}, P<0.001)$, there was no significant difference in the image quality $(P=0.91)$. Among patients with baseline $H R \geq 75 / \mathrm{min}$, the average HR tended to be lower in the high-dose landiolol group $(67.2 \pm 6.9 / \mathrm{min})$ compared with the low-dose landiolol group $(69.0 \pm 6.9 / \mathrm{min}, \mathrm{P}=0.10)$, and there was a corresponding difference in image quality between these two groups $(P=0.02)$. In conclusion, Although the decrease of HR was significantly larger in the propranolol group than in the landiolol groups, the image quality was similar. Among the patients who received landiolol, a higher dose was associated with a lower HR and better image quality. Further investigation to assess higher-dose bolus injection of landiolol or bolus injection following oral administration of a $\beta$-blocker would be needed.
\end{abstract}

Keywords: Cardiac computed tomography; Landiolol; Propranolol; Image quality; Coronary artery disease

\section{Background}

With the recent development of multidetector computed tomography (MDCT), cardiac computed tomography $(\mathrm{CCT})$ has become a reliable noninvasive diagnostic tool for coronary artery disease. (Achenbach et al. 2000; Nieman et al. 2001; Miller et al. 2008; Budoff et al. 2008; Meijboom et al. 2008; Raff et al. 2005). However, heart rate (HR) reduction is essential to achieve adequate image quality for diagnostic purposes, despite the recent advances in CT scanner hardware that have achieved a maximal rotation time of 270 to $350 \mathrm{msec}$. (Leschka et al. 2006; Shim et al. 2005; Muenzel et al. 2011; Korosoglou

\footnotetext{
* Correspondence: kyohei@yamaji.info

${ }^{\dagger}$ Equal contributors

'Division of Cardiology, Kokura Memorial Hospital, 3-2-1 Asano, Kokurakita-ku, Kitakyushu, Japan

Full list of author information is available at the end of the article
}

et al. 2010; Khan et al. 2011). Thus, either oral or intravenous $\beta$-blockers are routinely administered prior to CCT in order to obtain adequate images. (Gerber et al. 2003; Shapiro et al. 2008; Pannu et al. 2008; Degertekin et al. 2008; Roberts et al. 2009) Isobe et al. reported that continuous infusion of landiolol, a highly cardioselective and ultra-short acting $\beta$-blocker, was useful for CCT. (Isobe et al. 2008). Recently, Osawa et al. reported that bolus injection of landiolol following oral administration of metoprolol achieved sufficient reduction of the heart rate. (Osawa et al. 2013). Both of these initial studies included a relatively small number of patients. Therefore, we conducted a larger study to evaluate the efficacy of a single bolus injection of landiolol without prior oral premedication before CCT. The objective of the current 
study was to clarify whether the bolus injection of ultrashort acting $\beta$-blocker could improve the image quality or not.

\section{Methods}

\section{Study population}

A flow chart of the study is shown in Figure 1. A total of 1,094 consecutive patients who underwent CCT from December 1, 2011 to March 16, 2012 at our hospital were enrolled in this study. From December 1, 2011 to January 22, 2012 oral propranolol (Inderal, AstraZeneca K.K., Osaka, Japan) was administered before CCT (propranolol group), whereas intravenous landiolol hydrochloride (Corebeta, Ono Pharmaceutical Co., Osaka, Japan) was injected before CCT from January 23, 2012 to March 16, 2012. The patients who received landiolol were divided into two groups: one was given a low dose of landiolol from January 23 to February 18 (low-dose landiolol group) and the other was given a high dose of landiolol from February 19 to March 16 (high-dose landiolol group). Exclusion criteria were as follows: CCT for evaluation of bypass grafts or the ascending aorta, HR before administration $<60 / \mathrm{min}$ or $>90 / \mathrm{min}$, atrial fibrillation, implanted pacemaker, a history of vasospastic angina, advanced atrioventricular block, left ventricular ejection fraction $<40 \%$, systolic blood pressure $<90 \mathrm{mmHg}$, and known drug allergy. During the enrollment period for the propranolol group, patients who had a history of bronchial asthma were also excluded. All patients gave written informed consent and this study was approved by the hospital's institutional ethics committee.

\section{Landiolol groups}

Landiolol was injected as an intravenous bolus after initial scanning in the supine position and CCT was performed from 3 to 5 minutes later. A bolus dose of $0.125 \mathrm{mg} / \mathrm{kg}$ of landiolol was administered to patients in the low-dose landiolol group. In the high-dose landiolol group, the same dose of landiolol was administered to patients with a baseline $\mathrm{HR}<75 / \min (\mathrm{n}=121,56.8 \%)$, while both $0.125 \mathrm{mg} / \mathrm{kg}$ and an additional $3.75 \mathrm{mg}$ of landiolol were administered to patients with an $\mathrm{HR} \geq 75$ / $\min (n=92,43.2 \%)$. The total dose of landiolol was limited to $12.5 \mathrm{mg}$ in both groups. The low-dose and highdose landiolol groups were analyzed on an intention-totreat basis.

\section{Propranolol group}

In the propranolol group, premedication with oral propranolol $(10 \mathrm{mg})$ was given at 1.5 hours before CCT for patients with a body weight $<70 \mathrm{~kg}$, while $20 \mathrm{mg}$ was administered for patients with a body weight $\geq 70 \mathrm{~kg}$. Additional intravenous administration of $\beta$-blockers was prohibited by the study protocol.

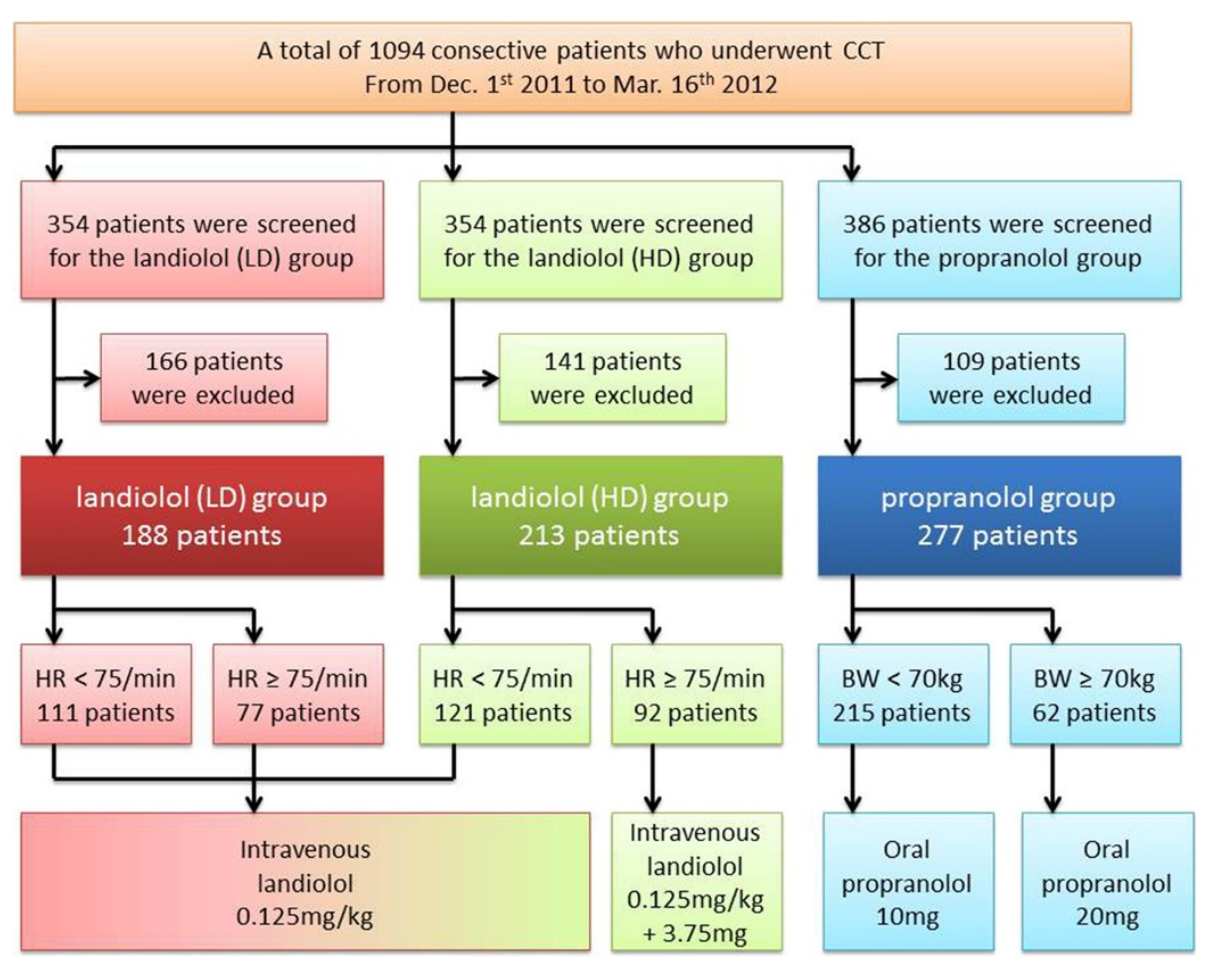

Figure 1 Study flow chart. There was a low-dose landiolol group, a high-dose landiolol group, and a propranolol group. CCT, cardiac computed tomography; LD, low dose; HD, high dose; HR, heart rate; BW, body weight. 


\section{CT scanning and post-processing}

Patients received premedication with nitroglycerin at $0.3 \mathrm{mg}$ sublingually, unless this was contraindicated. Scanning was performed using a 64-row MDCT scanner (Light Speed VCT, GE Healthcare. Waukesha, Wisconsin) with $64 \times 0.625-\mathrm{mm}$ collimation and a gantry rotation time of $350 \mathrm{msec}$. The tube current was modulated with a maximum of 550 to $700 \mathrm{~mA}$ (depending on the patient's size) during the period between $40 \%$ and $80 \%$ of the R-R interval and reduction by $80 \%$ during the remainder of the cardiac cycle, while the tube voltage was fixed at $120 \mathrm{kV}$. The helical pitch was selected just before scanning from 0.16 to 0.24 depending on the heart rate: helical pitch of 0.16 for patients with $\mathrm{HR}<36 / \mathrm{min}, 0.18$ for $\mathrm{HR}<40 / \mathrm{min}, 0.20$ for $\mathrm{HR}<44 / \mathrm{min}, 0.22$ for $\mathrm{HR}<48 / \mathrm{min}, 0.24$ for $\mathrm{HR}<62 / \mathrm{min}$, and 0.16 for $H R \geq 62 / \mathrm{min}$. A lower helical pitch was selected in patients who were likely to have premature beats during the main scan, whereas a higher pitch was selected in young patients and patients who had difficulty with breathholding. Final selection of the helical pitch was at the discretion of the radiologist. A test bolus of iohexol (Omnipaque $350350 \mathrm{mgI} / \mathrm{ml}$, Daiichi Sankyo, Tokyo, Japan) or iopamidol (Iopamiron $370370 \mathrm{mgI} / \mathrm{ml}$, Bayer Yakuhin, Osaka, Japan) was administered at a rate of $2.4 \mathrm{ml} / \mathrm{sec}$ to $5.0 \mathrm{ml} / \mathrm{sec}$ to ascertain the optimum rate and timing of injection for the main scan. Then $30 \mathrm{ml}$ to $80 \mathrm{ml}$ of contrast medium was injected intravenously during the main scan and a saline bolus $(20 \mathrm{ml})$ was injected intravenously immediately after the contrast agent (double-bolus protocol). For post-processing, the optimum time window was selected for each coronary vessel from either mid-diastole $(70,75$, or $80 \%$ ) of the cardiac cycle, or end-systole $(40,45$, or $50 \%)$. The "segment" and "burst" algorithms were used for half-scan reconstruction and multisector reconstruction, respectively. The optimal reconstruction algorithm was selected by viewing all images reconstructed with both algorithms side by side. The average and range of the HR during the main scan, total radiation exposure, main scan duration, occurrence of premature beats, and failure to maintain breathholding during the scan were recorded.

\section{Data analysis}

Assessment of the image quality score for each coronary vessel was done by two independent radiologists in a blinded manner regarding the clinical background using a previously reported five-point scale. (Leschka et al. 2006; Shim et al. 2005). (1) no motion artifacts and clear delineation of the segment; (2) minor artifacts and mild blurring of the segment; (3) moderate artifacts and moderate blurring without discontinuity; (4) severe artifacts and overlap or discontinuity of the segment; and (5) image not evaluable and vascular structures indistinguishable (Figure 2). The image quality score for a patient was defined as the maximum image quality score among the vessels.

\section{Statistical analysis}

Categorical variables and numerical variables were expressed as numbers and percentages. Frequency analysis was performed with the $\chi^{2}$ test. Variables such as the image quality score were compared using the KruskalWallis test. Normality of distribution was tested with the Shapiro-Wilk test. Continuous variables were expressed as the mean $\pm \mathrm{SD}$ and were compared by the unpaired $t$-test. To compare patient characteristics among the 3 groups, one-way analysis of variance was used. Variation in the decrease of HR was assessed by the Bartlett's test. All statistical analyses were performed using JMP 9.03 software (SAS Institute, Cary, NC). All reported P values are twosided and $\mathrm{P}<0.05$ was regarded as statistically significant.

\section{Results}

From a total of 1,094 consecutive patients who underwent CCT, 188 patients, 213 patients, and 277 patients were enrolled in the low-dose landiolol group, the highdose landiolol group, and the propranolol group, respectively, while 416 patients met the exclusion criteria and were excluded as shown in Table 1 . There was no significant difference in age, gender, height, weight, body mass index, and HR before $\beta$-blocker administration among the groups (Table 2).

Before $\beta$-blocker administration, the HR was $>75 / \mathrm{min}$ in $77(41.0 \%)$ patients from the low-dose landiolol group and $92(43.2 \%)$ patients from the high-dose landiolol group. The mean doses of landiolol in the low-dose and high-dose landiolol groups were $7.7 \pm 1.4 \mathrm{mg}$ and $9.2 \pm$ $2.2 \mathrm{mg}$, respectively. As the landiolol dose was limited up to12.5 mg, 2 patient with low-dose group and 7 patients with high-dose group received $1.75 \pm 0.18 \mathrm{mg}$ and $1.36 \pm 0.78 \mathrm{mg}$ fewer than prespecified dose, respectively. In the propranolol group, $10 \mathrm{mg}$ of propranolol was administered to 215 patients $(77.6 \%)$ and a dose of $20 \mathrm{mg}$ was administered to 62 patients (22.4\%) (Figure 1).

\section{Low-dose landiolol versus propranolol}

Although the average HR during the main scan was significantly lower in the propranolol group $(61.6 \pm 8.0$ / $\min )$ compared with that in the patients receiving lowdose landiolol $(64.1 \pm 7.4 / \mathrm{min}, \mathrm{P}<0.001)$, the maximum image quality score was similar between the patients given propranolol (mean $2.43 \pm 1.27$ ) and those given low-dose landiolol (mean 2.54 $\pm 1.31, \mathrm{P}=0.91$ ) (Table 3 and Figure 3). Regarding CT scanning parameters, a higher helical pitch was selected in the propranolol group $(\mathrm{P}<0.001)$. Consequently, multisector reconstruction was employed in $49.1 \%$ of the patients from the propranolol group versus $67.7 \%$ of those receiving low- 


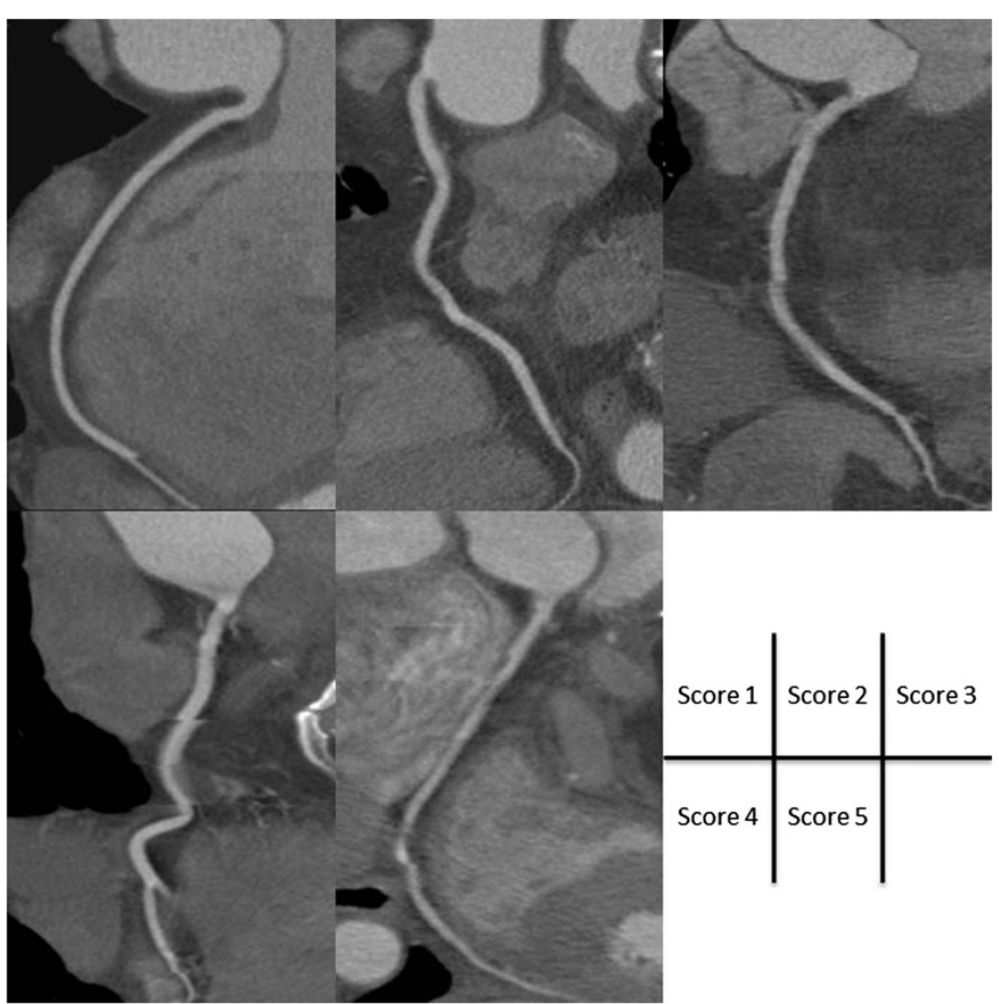

Figure 2 Curved multiplanar reconstruction images obtained by CCT. Examples of images given each score on five-point scale.

\section{Table 1 Exclusion criteria}

\begin{tabular}{|c|c|c|c|}
\hline & $\begin{array}{l}\text { Low-dose } \\
\text { landiolol }\end{array}$ & $\begin{array}{l}\text { High-dose } \\
\text { landiolol }\end{array}$ & Propranolol \\
\hline & $\begin{array}{c}n=166 / \\
354\end{array}$ & $\begin{array}{c}n=141 / \\
354\end{array}$ & $n=109 / 386$ \\
\hline $\begin{array}{l}\text { CCT for evaluating bypass } \\
\text { graft or ascending aorta }\end{array}$ & $51(14.4 \%)$ & $40(11.3 \%)$ & 19 (4.9\%) \\
\hline $\begin{array}{l}\mathrm{HR}<60 / \text { min before } \\
\text { administration }\end{array}$ & $69(19.5 \%)$ & $59(16.7 \%)$ & $37(9.6 \%)$ \\
\hline $\begin{array}{l}\mathrm{HR}>90 / \text { min before } \\
\text { administration }\end{array}$ & $16(4.5 \%)$ & $24(6.8 \%)$ & $18(4.7 \%)$ \\
\hline Atrial fibrillation & $14(4.0 \%)$ & $14(4.0 \%)$ & $9(2.3 \%)$ \\
\hline Permanent pacemaker & $7(2.0 \%)$ & $3(0.8 \%)$ & $3(0.8 \%)$ \\
\hline History of vasospastic angina & $2(0.6 \%)$ & $0(0.0 \%)$ & $1(0.3 \%)$ \\
\hline $\begin{array}{l}\text { Advanced atrioventricular } \\
\text { block }\end{array}$ & $2(0.6 \%)$ & $1(0.3 \%)$ & $0(0.0 \%)$ \\
\hline History of asthma & $0(0.0 \%)$ & $0(0.0 \%)$ & $20(5.2 \%)$ \\
\hline LVEF $<40 \%$ & $3(0.8 \%)$ & $0(0.0 \%)$ & $0(0.0 \%)$ \\
\hline $\begin{array}{l}\text { Hypotension before } \\
\text { administration (systolic } \\
\text { BP }<90 \mathrm{mmHg} \text { ) }\end{array}$ & $2(0.6 \%)$ & $0(0.0 \%)$ & $1(0.3 \%)$ \\
\hline Known drug allergy & $0(0.0 \%)$ & $0(0.0 \%)$ & $1(0.3 \%)$ \\
\hline
\end{tabular}

$\mathrm{CCT}$, cardiac computed tomography; HR, heart rate; LVEF, left ventricular ejection fraction; $\mathrm{BP}$, blood pressure. dose landiolol. The total radiation dose was smaller in the propranolol group compared with that in the patients receiving low-dose landiolol (1,343 \pm 402 DLP versus $1,450 \pm 335$ DLP, $\mathrm{P}=0.003)$. The decrease of HR was significantly larger in the propranolol group (11.0 \pm 7.2 / min) compared with that in the patients receiving lowdose landiolol $(8.8 \pm 5.7 / \mathrm{min})(\mathrm{P}<0.001)$. However, the

Table 2 Patient characteristics

\begin{tabular}{|c|c|c|c|c|}
\hline & $\begin{array}{l}\text { Low-dose } \\
\text { landiolol } \\
(n=188)\end{array}$ & $\begin{array}{l}\text { High-dose } \\
\text { landiolol } \\
(\mathrm{n}=213)\end{array}$ & $\begin{array}{l}\text { Propranolol } \\
\qquad(n=277)\end{array}$ & $P$ value \\
\hline Age & $67.6 \pm 10.9$ & $67.7 \pm 11.1$ & $67.0 \pm 11.9$ & 0.76 \\
\hline Male gender & 104 (55.3\%) & 120 (56.3\%) & 155 (56.0\%) & 0.98 \\
\hline Height, $\mathrm{cm}$ & $159.3 \pm 8.5$ & $159.3 \pm 9.2$ & $159.7 \pm 9.2$ & 0.90 \\
\hline Weight, kg & $60.6 \pm 11.3$ & $60.5 \pm 11.9$ & $61.4 \pm 12.9$ & 0.67 \\
\hline$<70 \mathrm{~kg}$ & 149 (79.3\%) & 168 (78.9\%) & 215 (77.6\%) & 0.90 \\
\hline$\geq 70 \mathrm{~kg}$ & 39 (20.7\%) & 45 (21.1\%) & 62 (22.4\%) & \\
\hline Body mass index & $23.8 \pm 3.3$ & $23.7 \pm 3.4$ & $23.9 \pm 3.8$ & 0.73 \\
\hline $\begin{array}{l}\text { HR before } \beta- \\
\text { blocker, } \text { min }^{-1}\end{array}$ & $72.9 \pm 7.3$ & $72.1 \pm 7.7$ & $72.5 \pm 7.7$ & 0.61 \\
\hline$<75 /$ min & $111(59.0 \%)$ & 121 (56.8\%) & $174(62.8 \%)$ & 0.39 \\
\hline$\geq 75 / \mathrm{min}$ & 77 (41.0\%) & 92 (43.2\%) & 103 (37.2\%) & \\
\hline
\end{tabular}

$\mathrm{HR}$, heart rate. 
Table 3 Heart rate parameters, CCT scan parameters, and image quality in patients receiving low-dose landiolol or propranolol

\begin{tabular}{|c|c|c|c|}
\hline & $\begin{array}{l}\text { Low-dose landiolol } \\
\qquad(n=188)\end{array}$ & $\begin{array}{l}\text { Propranolol } \\
(n=277)\end{array}$ & $P$ value \\
\hline $\begin{array}{l}\text { HR before } \beta \text {-blocker, } \\
\text { min }^{-1}\end{array}$ & $72.9 \pm 7.3$ & $72.5 \pm 7.7$ & 0.64 \\
\hline $\begin{array}{l}\text { Average } \mathrm{HR} \text { during the } \\
\text { main scan, } \mathrm{min}^{-1}\end{array}$ & $64.1 \pm 7.4$ & $61.6 \pm 8.0$ & $<0.001$ \\
\hline $\begin{array}{l}\text { Range of } \mathrm{HR} \text { during the } \\
\text { main scan, } \min ^{-1}\end{array}$ & $5.9 \pm 14.3$ & $4.4 \pm 10.1$ & 0.19 \\
\hline Decrease of $\mathrm{HR}$, $\mathrm{min}^{-1}$ & $8.8 \pm 5.7$ & $11.0 \pm 7.2$ & $<0.001$ \\
\hline $\begin{array}{l}\text { Time between } \beta \text {-blocker } \\
\text { administration and } \\
\text { main scan, min }\end{array}$ & $4.0 \pm 0.6$ & $90.5 \pm 16.0$ & $<0.001$ \\
\hline Helical pitch & & & $<0.001$ \\
\hline Step and shoot & $0(0.0 \%)$ & $5(1.8 \%)$ & \\
\hline 0.16 & $138(73.4 \%)$ & 141 (50.9\%) & \\
\hline 0.18 & $12(6.4 \%)$ & $26(9.4 \%)$ & \\
\hline 0.2 & $0(0.0 \%)$ & $3(1.1 \%)$ & \\
\hline 0.22 & $4(2.1 \%)$ & $22(7.9 \%)$ & \\
\hline 0.24 & $34(18.1 \%)$ & 80 (28.9\%) & \\
\hline No. of sectors used & & & $<0.001$ \\
\hline 1 & $61(32.5 \%)$ & 141 (50.9\%) & \\
\hline 2 & $119(63.3 \%)$ & $125(45.1 \%)$ & \\
\hline 3 & $8(4.4 \%)$ & $11(4.0 \%)$ & \\
\hline Total exposure, DLP & $1450 \pm 335$ & $1343 \pm 402$ & 0.003 \\
\hline Main scan duration, sec & $6.8 \pm 1.1$ & $6.4 \pm 1.3$ & $<0.001$ \\
\hline $\begin{array}{l}\text { Premature beats during } \\
\text { the main scan }\end{array}$ & $16(8.5 \%)$ & $15(5.4 \%)$ & 0.19 \\
\hline Failure at breathholding & $3(1.6 \%)$ & $9(3.2 \%)$ & 0.27 \\
\hline $\begin{array}{l}\text { Presence of coronary } \\
\text { artery plaque }\end{array}$ & $106(56.3 \%)$ & $156(56.3 \%)$ & 0.99 \\
\hline Presence of calcification & 139 (73.9\%) & 199 (71.8\%) & 0.62 \\
\hline $\begin{array}{l}\text { Maximum image quality } \\
\text { score per patient }\end{array}$ & & & 0.91 \\
\hline 1 & $55(29.3 \%)$ & $88(31.8 \%)$ & \\
\hline 2 & $42(22.3 \%)$ & $63(22.7 \%)$ & \\
\hline 3 & $44(23.4 \%)$ & $64(23.1 \%)$ & \\
\hline 4 & 29 (15.4\%) & 42 (15.2\%) & \\
\hline 5 & $18(9.6 \%)$ & $20(7.2 \%)$ & \\
\hline \multicolumn{4}{|l|}{$\begin{array}{l}\text { Image quality score } \\
\text { per vessel }\end{array}$} \\
\hline RCA & & & 0.86 \\
\hline 1 & 67 (35.6\%) & 106 (38.3\%) & \\
\hline 2 & $40(21.3 \%)$ & 66 (23.8\%) & \\
\hline 3 & 38 (20.2\%) & $50(18.1 \%)$ & \\
\hline 4 & 31 (16.5\%) & 40 (14.4\%) & \\
\hline 5 & $12(6.4 \%)$ & 15 (5.4\%) & \\
\hline
\end{tabular}

Table 3 Heart rate parameters, CCT scan parameters, and image quality in patients receiving low-dose landiolol or propranolol (Continued)

\begin{tabular}{cccc}
\hline LAD & & & 0.70 \\
1 & $87(46.3 \%)$ & $132(47.7 \%)$ & \\
2 & $42(22.3 \%)$ & $72(26.0 \%)$ & \\
3 & $38(20.2 \%)$ & $45(16.2 \%)$ & \\
4 & $16(8.5 \%)$ & $19(6.9 \%)$ & \\
5 & $5(2.7 \%)$ & $9(3.2 \%)$ & \\
LCX & & & 0.32 \\
1 & $82(43.6 \%)$ & $129(46.6 \%)$ & \\
2 & $44(23.4 \%)$ & $69(24.9 \%)$ & \\
3 & $31(16.5 \%)$ & $49(17.7 \%)$ & \\
4 & $24(12.8 \%)$ & $19(6.9 \%)$ & \\
5 & $7(3.7 \%)$ & $11(4.0 \%)$ & \\
\hline
\end{tabular}

$\mathrm{CCT}$, cardiac computed tomography; HR, heart rate; DLP, dose-length product; $R C A$, right coronary artery; $L A D$, left anterior descending coronary artery; $L C X$, left circumflex artery.

variance of the decrease in HR was significantly larger in the propranolol group, as shown in Figure 4 (32.5 versus 51.8, $\mathrm{P}<0.001)$. In this study, we assessed $\mathrm{HR}$ variability during the scan from the range of HR values, and found no significant difference in the range of HR during the main scan between patients treated with low-dose landiolol group and those given propranolol.

\section{Low-dose versus high-dose landiolol}

Among patients with a baseline HR $\geq 75 / \min$ (77 [41.0\%] patients in the low-dose landiolol group and 92 [43.2\%] patients in the high-dose landiolol group), the average HR during the main scan was tended to be lower in the highdose landiolol group $(67.2 \pm 6.9 / \mathrm{min})$ compared with that in the low-dose landiolol group $(69.0 \pm 6.9 / \mathrm{min}, \mathrm{P}=0.10)$, and there was a corresponding difference of the maximum image quality score between the high-dose landiolol group (mean $2.53 \pm 1.21$ ) and low-dose landiolol group (mean $3.04 \pm 1.41, \mathrm{P}=0.02$ ) (Table 4 and Figure 3 ).

Possible side effects of $\beta$-blockers were reported in two patients from the low-dose landiolol group: One patient had sinus bradycardia (40/minute) with frequent ventricular premature beats that recovered after $5 \mathrm{mi}-$ nutes, and the other showed a decrease of blood pressure from $159 / 95 \mathrm{mmHg}$ to $86 / 55 \mathrm{mmHg}$ that returned to $114 / 78 \mathrm{mmHg}$ after 10 minutes. Both patients were asymptomatic.

\section{Discussion}

The objective of the current study was to clarify whether the bolus injection of ultra-short acting $\beta$-blocker could improve the image quality or not. The main findings of this study were as follows: (1) Image quality was similar 
between propranolol and low-dose landiolol, although the decrease in HR was significantly larger in the propranolol group than in the low-dose landiolol groups. (2) In the patients receiving landiolol with a baseline $H R \geq 75$ / min, the higher dose was associated with a lower HR and better image quality. (3) Variance in the decrease of HR was significantly smaller and the mean time from administration to performing the main scan was significantly shorter in the landiolol groups.

Despite promising advances in MDCT, heart rate reduction is still essential for CCT. (Leschka et al. 2006; Shim et al. 2005; Muenzel et al. 2011; Korosoglou et al. 2010; Khan et al. 2011). We compared a single bolus injection of landiolol with oral administration of propranolol, and demonstrated a significantly greater decrease of HR in the propranolol group without improvement of image quality. This might be partly explained by the higher frequency of multisector reconstruction with the slight increase of total exposure in the landiolol groups. With a gantry rotation speed of $350 \mathrm{msec}$, an HR of 62,68 , or $78 / \mathrm{min}$ becomes the cut-off value between half-scan reconstruction and multisector reconstruction when the helical pitch is 0.16 ,

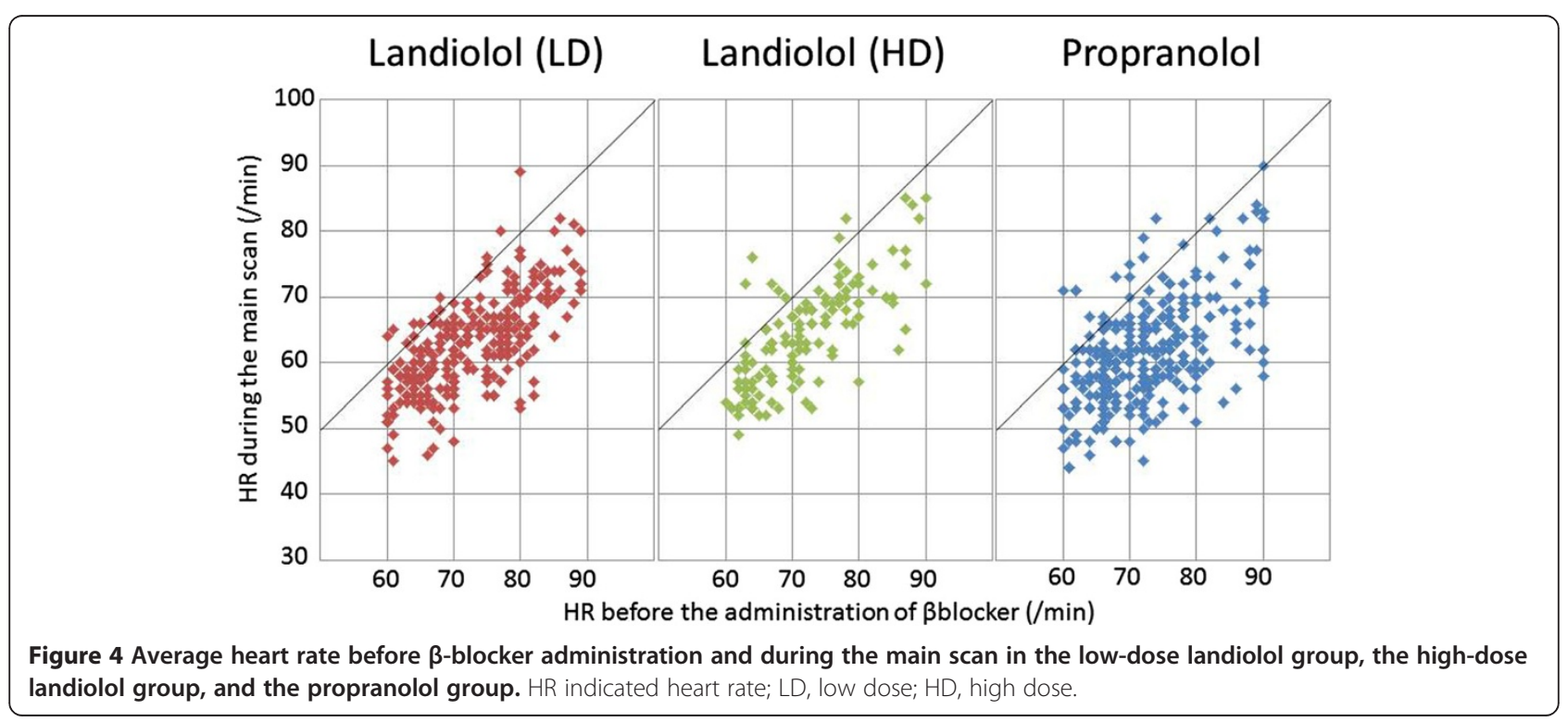


Table 4 Heart rate parameters, CCT scan parameters, and image quality in the low-dose landiolol group and the high-dose landiolol group among patients with baseline $H R \geq 75 / \mathrm{min}$

\begin{tabular}{|c|c|c|c|}
\hline & $\begin{array}{l}\text { Low-dose } \\
\text { landiolol } \\
(n=77)\end{array}$ & $\begin{array}{c}\text { High-dose } \\
\text { landiolol } \\
(\mathrm{n}=92)\end{array}$ & $P$ value \\
\hline $\begin{array}{l}\text { HR before } \beta \text {-blocker, } \\
\text { min }^{-1}\end{array}$ & $80.3 \pm 4.3$ & $79.7 \pm 4.1$ & 0.42 \\
\hline $\begin{array}{l}\text { Average } H R \text { during the } \\
\text { main scan, } \text { min }^{-1}\end{array}$ & $69.0 \pm 6.9$ & $67.2 \pm 6.9$ & 0.10 \\
\hline $\begin{array}{l}\text { Range of HR during the } \\
\text { main scan, } \text { min }^{-1}\end{array}$ & $5.3 \pm 12.2$ & $5.9 \pm 14.9$ & 0.77 \\
\hline Decrease at $\mathrm{HR}, \min ^{-1}$ & $11.2 \pm 6.0$ & $12.5 \pm 6.1$ & 0.18 \\
\hline $\begin{array}{l}\text { Time between } \beta \text {-blocker } \\
\text { administration and main } \\
\text { scan, min }\end{array}$ & $4.1 \pm 0.66$ & $3.7 \pm 0.80$ & $<0.001$ \\
\hline Helical pitch & & & 0.69 \\
\hline Step and shoot & $0(0.0 \%)$ & $0(0.0 \%)$ & \\
\hline 0.16 & $72(93.5 \%)$ & $86(93.5 \%)$ & \\
\hline 0.18 & $3(3.9 \%)$ & $5(5.4 \%)$ & \\
\hline 0.2 & $0(0.0 \%)$ & $0(0.0 \%)$ & \\
\hline 0.22 & $0(0.0 \%)$ & $0(0.0 \%)$ & \\
\hline 0.24 & $2(2.6 \%)$ & $1(1.1 \%)$ & \\
\hline No. of sectors used & & & 0.27 \\
\hline 1 & $10(13.0 \%)$ & $6(6.5 \%)$ & \\
\hline 2 & $59(76.6 \%)$ & 79 (85.9\%) & \\
\hline 3 & $8(10.4 \%)$ & $7(7.6 \%)$ & \\
\hline Total exposure, DLP & $1582 \pm 212$ & $1594 \pm 232$ & 0.74 \\
\hline Main scan duration, sec & $7.2 \pm 0.63$ & $7.5 \pm 0.97$ & 0.01 \\
\hline $\begin{array}{l}\text { Premature beats during } \\
\text { the main scan }\end{array}$ & $6(7.8 \%)$ & $6(6.5 \%)$ & 0.75 \\
\hline Failure at breathholding & $1(1.3 \%)$ & $2(2.2 \%)$ & 0.67 \\
\hline $\begin{array}{l}\text { Presence of coronary } \\
\text { artery plaque }\end{array}$ & $32(41.6 \%)$ & $51(55.4 \%)$ & 0.07 \\
\hline Presence of calcification & $53(68.3 \%)$ & $73(79.4 \%)$ & 0.12 \\
\hline $\begin{array}{l}\text { Maximum image quality } \\
\text { score per patient }\end{array}$ & & & 0.02 \\
\hline 1 & 15 (19.5\%) & $21(22.8 \%)$ & \\
\hline 2 & $14(18.2 \%)$ & $28(30.4 \%)$ & \\
\hline 3 & $16(20.8 \%)$ & $23(25.0 \%)$ & \\
\hline 4 & 17 (22.1\%) & $13(14.1 \%)$ & \\
\hline 5 & 15 (19.5\%) & 7 (7.6\%) & \\
\hline \multicolumn{4}{|l|}{$\begin{array}{l}\text { Image quality score per } \\
\text { vessel }\end{array}$} \\
\hline RCA & & & 0.005 \\
\hline 1 & 19 (24.7\%) & 30 (32.6\%) & \\
\hline 2 & 11 (14.3\%) & 26 (28.3\%) & \\
\hline 3 & 16 (20.8\%) & 20 (21.7\%) & \\
\hline
\end{tabular}

Table 4 Heart rate parameters, CCT scan parameters, and image quality in the low-dose landiolol group and the high-dose landiolol group among patients with baseline HR $\geq 75 /$ min (Continued)

\begin{tabular}{cccc}
\hline 4 & $21(27.3 \%)$ & $10(10.9 \%)$ & \\
5 & $10(13.0 \%)$ & $6(6.5 \%)$ & \\
LAD & & & 0.054 \\
1 & $29(37.7 \%)$ & $39(42.4 \%)$ & \\
2 & $13(16.9 \%)$ & $32(34.8 \%)$ & \\
3 & $19(24.7 \%)$ & $11(12.0 \%)$ & \\
4 & $13(16.9 \%)$ & $8(8.7 \%)$ & \\
5 & $3(3.9 \%)$ & $2(2.2 \%)$ & \\
LCX & & & 0.02 \\
1 & $29(37.7 \%)$ & $44(47.8 \%)$ & \\
2 & $12(15.6 \%)$ & $20(21.7 \%)$ & \\
3 & $10(13.0 \%)$ & $17(18.5 \%)$ & \\
4 & $19(24.7 \%)$ & $8(8.7 \%)$ & \\
5 & $7(9.1 \%)$ & $3(3.3 \%)$ & \\
\hline
\end{tabular}

$\mathrm{CCT}$, cardiac computed tomography; $\mathrm{HR}$, heart rate; DLP, dose-length product; $R C A$, right coronary artery; $L A D$, left anterior descending coronary artery; $L C X$, left circumflex artery.

0.18, and 0.20, respectively. (Ohnesorge et al. 2000; Flohr and Ohnesorge 2001). The current study included patients with an HR of around 60/min at the time of the main scan, and there was a significant difference in the performance of multisector reconstruction that might have improved image quality in the patients receiving low-dose landiolol, despite the small absolute difference of $2.5 / \mathrm{min}$ for the HR during the main scan.

Leschka et al. reported that not only the absolute reduction of HR but also a decrease of HR variability is important for achieving adequate image quality. (Leschka et al. 2006). The variance in the decrease of HR was smaller in the landiolol groups compared with the propranolol group along with the shorter time between $\beta$ blocker administration and performance of the main scan. We needed to avoid specific heart rates synchronized with gantry rotation, as we were unable to perform multisector reconstruction if the cardiac cycle length was an exact multiple of the gantry rotation speed. For example, if the patient had an HR of 86 (cardiac cycle length $=700 \mathrm{msec}$ ) during the main scan, we could not perform multisector reconstruction when the gantry rotation speed was $350 \mathrm{Msec}$. A protocol that involves continuous infusion with stepwise dose increments might be more beneficial for controlling the HR prior to CCT. However, the current study demonstrated that an additional bolus dose could also reduce the HR and improve image quality without any complications. Further investigation to assess higher-dose bolus injection alone and bolus injection 


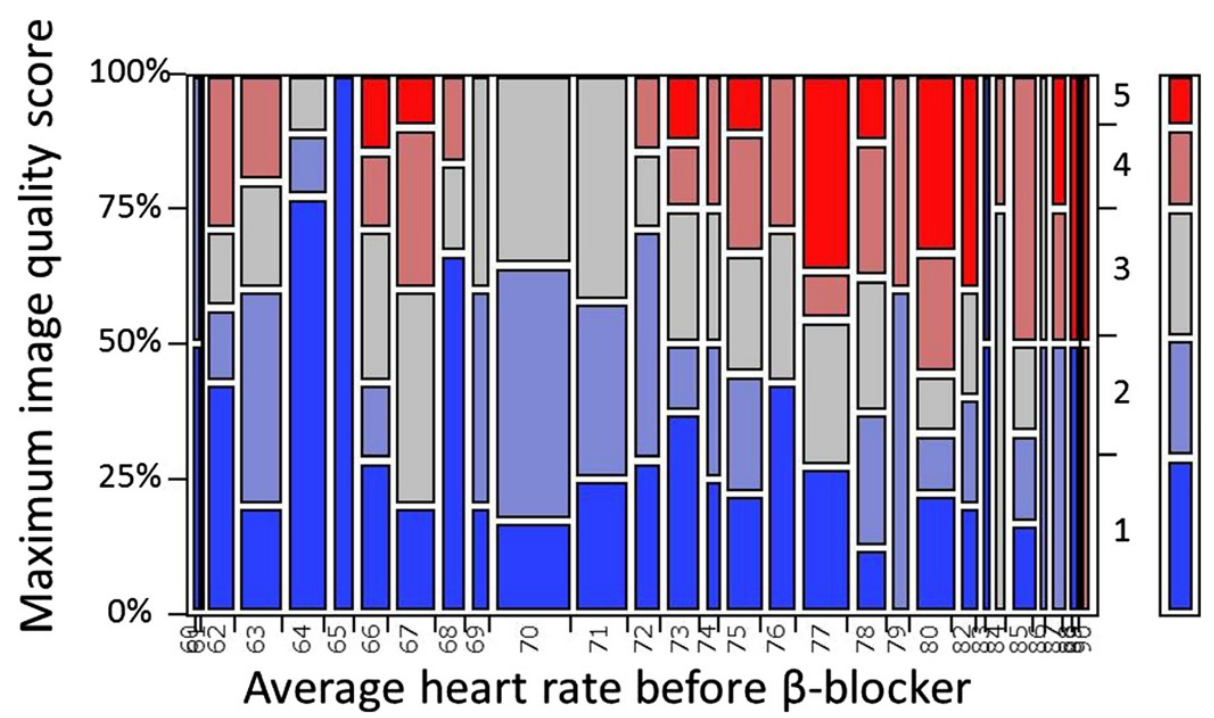

Figure 5 Mosaic plot for maximum image quality socre and average heart rate before $\beta$-blocker in patients with low-dose landiolol group.

following oral administration of a $\beta$-blocker would be needed to clarify the efficacy of bolus landiolol compared with a stepwise continuous infusion protocol.

In this study, two patients developed suspected side effects after bolus injection of landiolol, including transient sinus bradycardia and severe hypotension. Owing to the short half-life of this agent, (Atarashi et al. 2000). The adverse effects were not prolonged and resolved completely. De Graaf et al. reported that contraindications to oral $\beta$-blocker therapy exist in a substantial proportion of patients undergoing CCT, (De Graaf et al. 2010). although administration of $\beta$-blocker was essential for better image quality. Because the absolute number of patients with adverse events was small, we could not assess the difference in the incidence of events between patients receiving bolus injection of landiolol and those given oral propranolol. However, landiolol is a highly $\beta 1$-selective and ultra-short acting $\beta$-blocker that is expected to be associated with less persistent adverse effects.

The present study has several limitations. First, the dose of landiolol was specified and additional injections were prohibited by the study protocol. Adjusting the dose of landiolol for each patient could have achieved the optimal HR and further improved image quality. Furthermore, a prespecified dose of $3.75 \mathrm{mg}$ and HR of $75 / \mathrm{min}$ would not be most effective cut off points. However, in low-dose landiolol group, patients with the baseline $H R \geq 75 / \mathrm{min}$ were prone to have poor image quality (maximum image score $\geq 4$ ) as compared with those with baseline $\mathrm{HR}<75 / \mathrm{min}$ as shown in Figure 5. Second, this was not a randomized trial, hence the results may be biased. Because the helical pitch was set at the discretion of the radiologist, there were substantial differences of the CT scanning parameters and post-processing. Although these parameters were mainly determined automatically based on the HR just before the main scan, the radiologists expected that the HR would change after scanning parameters were selected in the landiolol groups. Third, the number of patients was relatively small (especially for evaluating safety), even though more than 400 patients were treated with landiolol.Fourth, the low penetration rate of step and shoot mode use could increase the radiation dose. Furthermore, radiation dose did not decrease despite of injection of high dose landiolol. Using step and shoot technique could be more beneficial not only for better image quality but also for reducing radiation dose. Finally, we did not evaluate the other medications prior to the CCT except for the $\beta$-blocker based on the study protocol. Administrating the $\beta$-blocker could not be effective in patients with prior medication of cardiovascular drugs including $\beta$-blocker for coexisting cardiac disease.

\section{Conclusions}

Although the decrease of HR was significantly larger in the propranolol group than in the landiolol groups, the image quality was similar with the two $\beta$-blockers. Among the patients receiving landiolol, a higher dose was associated with a lower HR and better image quality. Further investigation to assess higher-dose bolus injection of landiolol or bolus injection following oral administration of a $\beta$-blocker would be needed. 


\section{Abbreviations}

CCT: Cardiac computed tomography; HR: Heart rate; MDCT: Multidetector computed tomography.

\section{Competing interests}

The authors declare that they have no competing interests.

\section{Authors' contributions}

$\mathrm{KY}$ and $\mathrm{YN}$ equally contributed to planning the study, data collection and analysis and drafting the manuscript. TS, ZM, and IY contrubuted to data collection. YS, SS, KA, and MN contributed to conception of the study. All authors read and approved the final manuscript.

\section{Author details}

'Division of Cardiology, Kokura Memorial Hospital, 3-2-1 Asano, Kokurakita-ku, Kitakyushu, Japan. ${ }^{2}$ Division of Radiological Technology, Kokura Memorial Hospital, 3-2-1 Asano, Kokurakita-ku, Kitakyushu, Japan.

\section{Received: 25 November 2013 Accepted: 28 January 2014}

Published: 17 February 2014

\section{References}

Achenbach S, Ulzheimer S, Baum U, Kachelriess M, Ropers D, Giesler T, Bautz W, Daniel WG, Kalender WA, Moshage W (2000) Noninvasive coronary angiography by retrospectively ECG-gated multislice spiral CT. Circulation 102:2823-2828

Atarashi H, Kuruma A, Yashima M, Saitoh H, Ino T, Endoh Y, Hayakawa H (2000) Pharmacokinetics of landiolol hydrochloride, a new ultra-short-acting betablocker, in patients with cardiac arrhythmias. Clin Pharmacol Ther 68:143-150

Budoff MJ, Dowe D, Jollis JG, Gitter M, Sutherland J, Halamert E, Scherer M, Bellinger R, Martin A, Benton R, Delago A, Min JK (2008) Diagnostic performance of 64-multidetector row coronary computed tomographic angiography for evaluation of coronary artery stenosis in individuals without known coronary artery disease: results from the prospective multicenter ACCURACY (Assessment by Coronary Computed Tomographic Angiography of Individuals Undergoing Invasive Coronary Angiography) trial. J Am Coll Cardiol 52:1724-1732

de Graaf FR, Schuijf JD, van Velzen JE, Kroft LJ, de Roos A, Sieders A, Jukema JW, Schalij MJ, van der Wall EE, Bax JJ (2010) Evaluation of contraindications and efficacy of oral Beta blockade before computed tomographic coronary angiography. Am J Cardiol 105:767-772

Degertekin M, Gemici G, Kaya Z, Bayrak F, Guneysu T, Sevinc D, Mutlu B, Aytaclar S (2008) Safety and efficacy of patient preparation with intravenous esmolol before 64-slice computed tomography coronary angiography. Coron Artery Dis 19:33-36

Flohr T, Ohnesorge B (2001) Heart rate adaptive optimization of spatial and temporal resolution for electrocardiogram-gated multislice spiral $C T$ of the heart. J Comput Assist Tomogr 25:907-923

Gerber TC, Kuzo RS, Lane GE, O'Brien PC, Karstaedt N, Morin RL, Safford RE, Blackshear JL, Pietan JH (2003) Image quality in a standardized algorithm for minimally invasive coronary angiography with multislice spiral computed tomography. J Comput Assist Tomogr 27:62-69

Isobe S, Sato K, Sugiura K, Mimura T, Kobayashi M, Meno C, Kato M, Ishii H, Murohara T (2008) Feasibility of intravenous administration of landiolol hydrochloride for multislice computed tomography coronary angiography: initial experience. Circ J 72:1814-1820

Khan A, Khosa F, Nasir K, Yassin A, Clouse ME (2011) Comparison of radiation dose and image quality: 320-MDCT versus 64-MDCT coronary angiography. AJR Am J Roentgenol 197:163-168

Korosoglou G, Mueller D, Lehrke S, Steen H, Hosch W, Heye T, Kauczor HU, Giannitsis E, Katus HA (2010) Quantitative assessment of stenosis severity and atherosclerotic plaque composition using 256-slice computed tomography. Eur Radiol 20:1841-1850

Leschka S, Wildermuth S, Boehm T, Desbiolles L, Husmann L, Plass A, Koepfli P, Schepis T, Marincek B, Kaufmann PA, Alkadhi H (2006) Noninvasive coronary angiography with 64-section $\mathrm{CT}$ : effect of average heart rate and heart rate variability on image quality. Radiology 241:378-385

Meijboom WB, Meijs MF, Schuijf JD, Cramer MJ, Mollet NR, van Mieghem CA Nieman K, van Werkhoven JM, Pundziute G, Weustink AC, de Vos AM, Pugliese F, Rensing B, Jukema JW, Bax JJ, Prokop M, Doevendans PA, Hunink MG, Krestin GP, de Feyter PJ (2008) Diagnostic accuracy of 64-slice computed tomography coronary angiography: a prospective, multicenter, multivendor study. J Am Coll Cardiol 52:2135-2144

Miller JM, Rochitte CE, Dewey M, Arbab-Zadeh A, Niinuma H, Gottlieb I, Paul N, Clouse ME, Shapiro EP, Hoe J, Lardo AC, Bush DE, de Roos A, Cox C, Brinker J, Lima JA (2008) Diagnostic performance of coronary angiography by 64-row CT. N Engl J Med 359:2324-2336

Muenzel D, Noel PB, Dorn F, Dobritz M, Rummeny EJ, Huber A (2011) Step and shoot coronary CT angiography using 256-slice CT: effect of heart rate and heart rate variability on image quality. Eur Radiol 21:2277-2284

Nieman K, Oudkerk M, Rensing BJ, van Ooijen P, Munne A, van Geuns RJ, de Feyter PJ (2001) Coronary angiography with multi-slice computed tomography. Lancet 357:599-603

Ohnesorge B, Flohr T, Becker C, Kopp AF, Schoepf UJ, Baum U, Knez A, Klingenbeck-Regn K, Reiser MF (2000) Cardiac imaging by means of electrocardiographically gated multisection spiral CT: initial experience. Radiology 217:564-571

Osawa K, Miyoshi T, Sato S, Akagi N, Morimitsu Y, Nakamura K, Kohno K, Kusano K, Kanazawa S, Ito H (2013) Safety and efficacy of a bolus injection of landiolol hydrochloride as a premedication for multidetector-Row computed tomography coronary angiography. Circ J 77:146-152

Pannu HK, Sullivan C, Lai S, Fishman EK (2008) Evaluation of the effectiveness of oral Beta-blockade in patients for coronary computed tomographic angiography. J Comput Assist Tomogr 32:247-251

Raff GL, Gallagher MJ, O'Neill WW, Goldstein JA (2005) Diagnostic accuracy of noninvasive coronary angiography using 64-slice spiral computed tomography. J Am Coll Cardiol 46:552-557

Roberts WT, Wright AR, Timmis JB, Timmis AD (2009) Safety and efficacy of a rate control protocol for cardiac CT. Br J Radiol 82:267-271

Shapiro MD, Pena AJ, Nichols JH, Worrell S, Bamberg F, Dannemann N, Abbara S, Cury RC, Brady TJ, Hoffmann U (2008) Efficacy of pre-scan beta-blockade and impact of heart rate on image quality in patients undergoing coronary multidetector computed tomography angiography. Eur J Radiol 66:37-41

Shim SS, Kim Y, Lim SM (2005) Improvement of image quality with beta-blocker premedication on ECG-gated 16-MDCT coronary angiography. AJR Am J Roentgenol 184:649-654

doi:10.1186/2193-1801-3-93

Cite this article as: Nakamura et al:: A comparison of bolus injection of landiolol versus oral administration of propranolol before cardiac computed tomography. SpringerPlus 2014 3:93.

\section{Submit your manuscript to a SpringerOpen ${ }^{\odot}$ journal and benefit from:}

- Convenient online submission

- Rigorous peer review

- Immediate publication on acceptance

- Open access: articles freely available online

- High visibility within the field

- Retaining the copyright to your article

Submit your next manuscript at $>$ springeropen.com 